\title{
Weaving of biomineralization framework in rotaliid foraminifera: implications for paleoceanographic proxies
}

\author{
Yukiko Nagai $^{1,2,3}$, Katsuyuki Uematsu ${ }^{4}$, Chong Chen ${ }^{3}$, Ryoji Wani ${ }^{5}$, Jarosław Tyszka ${ }^{6}$, and Takashi Toyofuku ${ }^{3,7}$ \\ ${ }^{1}$ Graduate School of Environment and Information Sciences, Yokohama National University, 79-7, Tokiwadai, \\ Hodogaya-ku, Yokohama, 240-8501, Japan \\ ${ }^{2}$ National Museum of Nature and Science, 4-1-1 Amakubo, Tsukuba-shi, Ibaraki 305-0005, Japan \\ ${ }^{3}$ Japan Agency for Marine-Earth Science and Technology (JAMSTEC), Natsushima-cho 2-15, Yokosuka, 237-0061, Japan \\ ${ }^{4}$ Marine Works Japan Ltd., 3-54-1 Oppama-higashi, Yokosuka 237-0063, Japan \\ ${ }^{5}$ Faculty of Environment and Information Sciences, Yokohama National University, 79-7, Tokiwadai, \\ Hodogaya-ku, Yokohama, 240-8501, Japan \\ ${ }^{6}$ ING PAN - Institute of Geological Sciences, Polish Academy of Sciences, Research Centre in Cracow, \\ Senacka 1, 31-002 Kraków, Poland \\ ${ }^{7}$ Tokyo University of Marine Science and Technology (TUMSAT), 4-5-7, Konan Minato-ku, Tokyo 108-8477, Japan
}

Correspondence: Yukiko Nagai (nagai.y@jamstec.go.jp)

Received: 20 June 2018 - Discussion started: 23 July 2018

Revised: 20 September 2018 - Accepted: 25 September 2018 - Published: 14 November 2018

\begin{abstract}
Elemental and/or isotopic signatures of calcareous tests of foraminifera are commonly used to reconstruct paleoenvironmental conditions. A major problem, often referred to as the "vital effect", is that such geochemical signatures stored in inorganic calcium carbonates differ greatly under the same environmental conditions, as well as between taxa, species, individuals, etc. This effect was previously explained by relative contributions between passive vs. active ion transport patterns, but their details are still under investigation. In this study, the functional role of pseudopodial structures during chamber formation is elucidated by detailed observation of Ammonia beccarii (Linnaeus, 1758) using a time-lapse optical imaging system and high-resolution electron microscopy. We document triple organic layers sandwiching carbonate precipitation sites for the first time. The three major organic layers (outer organic layer, primary organic sheet, and inner organic layer) are formed by an initial framework of pseudopodia overlaid with further layerlike pseudopodia. The primary organic sheet seems to facilitate early calcium carbonate nucleation, then entrapped by double precipitation sites. We further show that calcification starts when outer or inner organic layers still exhibit tiny gaps (holes within the framework) that may serve as pathways for passive ion exchange (e.g. $\mathrm{Mg}^{2+}$ ) between seawater and the
\end{abstract}

confined precipitation space. Nevertheless, the majority of wall thickening occurs when the precipitation site is completely isolated from seawater, which implies active ion exchange. This may explain the differences in $\mathrm{Mg} / \mathrm{Ca}$ ratios in early and later stages of calcification observed in previous studies. Our study provides insight into resolving a key "missing piece" in understanding foraminiferal calcification through culture experiments and in-depth observations of living animals. Our findings contribute to interpreting and understanding biogeochemical proxies by showing that the "vital effect", specifically elemental and isotopic ratios along chamber walls, is directly linked to spatio-temporal organization of the "biomineralization sandwich" controlled by the three major organic layers.

\section{Introduction}

The calcification process of the foraminiferal test is the phase of growth in which elemental and isotopic compositions of the test is determined and is also the key to generating their morphological diversity. In recent years, the foraminiferal test has become widely applied as a palaeoenvironmental proxy, and its geochemical and isotopic composition has be- 
come one of the major tools in palaeoenvironmental reconstructions, even though the test morphology and chemical composition depend to a certain extent on the environment (De Nooijer et al., 2014; Schiebel et al., 2017). To this end, elucidating the detailed mechanisms of foraminiferal calcification has been treated with great interest in the field of geosciences. For example, it has been proven by culturing experiments that the seawater temperature and the $\mathrm{Mg} / \mathrm{Ca}$ ratio of foraminifera show a strong linear correlation (Nürnberg et al., 1996; Toyofuku et al., 2000). Meanwhile, it is also known that the incorporation ratio of $\mathrm{Mg} / \mathrm{Ca}$ is variable and species specific (summary in Toyofuku et al., 2011). The chemical distributions, however, vary among even individuals of the same species and exhibit zonation, corresponding to the test wall structure (Kunioka et al., 2006; van Dijk et al., 2017). These variations in chemical composition, both inter- and intraspecific, are inclusively termed the "vital effect" (Urey, 1951). In order to reconstruct accurate palaeoenvironments, it is important to utilize reliable proxies, such as the chemistry and isotopic composition of foraminifera tests. Therefore, the biological processes of chamber formation is of great importance and interest.

Observation of the foraminiferal chamber formation process has been reported from as early as 1854 using the genus Peneroplis (Schultze, 1854), and many species have been documented thereafter (e.g. Myers, 1935, 1940, 1943; Jepps, 1942; Sliter, 1970; Berthold, 1976; Spindler and Röttger, 1973). Superfine structure observation by scanning and transmission electron microscopy (SEM and TEM) have been reported in order to carry out more detailed documentation of the cellular process of calcite precipitation during chamber formation in the benthic species Rosalina floridana (Angell, 1967) as well as the planktonic species Globorotalia truncatulinoides (Hemleben et al., 1986) and Orbulina universa (Spero, 1988). The common features summarized from these detailed observations on benthic and planktonic species point to the fact that cytoplasm and the many types of organic sheet-like structures (i.e. organic layers like outer organic layer, OOL, and inner organic layer, IOL) play fundamental roles in calcification, as opposed to simple chemical reactions between calcium and carbonate ions.

Pseudopodium is one of the key features of foraminiferal biology. Pseudopodia form a part of the cytoplasm consisting of cytoskeleton structures, such as microtubules and actin filaments, as well as other organelles like mitochondria, spherical structures, and vacuoles (Marszalek, 1969, reviewed in Travis and Bowser, 1991). Pseudopodium represents a multifunctional cellular structure serving various purposes such as locomotion, feeding, digestion, and chamber formation. Foraminiferal pseudopodia are usually named granuloreticulopodia (see Travis and Bowser, 1991) to define a granular reticulated pseudopodium responsible for feeding, digestion and locomotion; in the present paper we will simply use pseudopodia as it is a more general term. The appearance of pseudopodia changes during chamber formation and a fan- like array of pseudopodia develops (Bé et al., 1979). Then, an organic structure that forms the framework for chamber formation, called "anlage", is formed (Angell, 1967). In benthic foraminifers, an algal cyst composed of foreign detritus and other materials is constructed around this anlage (Angell, 1967). Anlage is largely constructed by foamy and spherical microstructures $(<1 \mu \mathrm{m})$ (Angel, 1967; Hemleben et al., 1986) and is bulging in shape, which led some authors to call it the "bulge" in early studies using planktonic foraminifers (e.g. Bé et al., 1979). This bulging anlage is the three-dimensional structure that becomes the precursor of the chamber. There are three organic layers in the anlage: the one on the outer surface has been termed the OOL (Spero, 1988), the one in the middle was initially named the "primary organic membrane" (POM) (Hemleben et al., 1986) but later changed to "primary organic sheet" (POS) (Erez, 2003), and the innermost one is called the IOL (Spero, 1988). Precipitation of calcium carbonate microcrystals takes place on both sides of the POS, sandwiched between the outer and inner organic layers. In addition to these three organic layers, the term anlage is now loosely accepted to include the numerous pseudopodial cytoplasm that are present around them during calcification. Since different authors have different views and definitions as to what anlage means (e.g. Angell, 1979; Bé et al., 1979; Hemleben et al., 1986), hereafter we refrain from using the term anlage and instead use 'organic scaffolding' to refer to the organic framework that the chamber wall is built upon.

In order to investigate the fundamental functions of the POS, the OOL and the IOL during chamber formation, Nagai et al. (2018) conducted focused ion-beam (FIB) processing on a foraminifera specimen during calcification, which allows the thin-sectioning of the site of calcification (SOC) without decalcification to observe cytoplasm and well-preserved morphology of the calcifying test (calcium carbonate crystals) together using SEM/TEM. Their observations clearly show that the organic scaffolding has numerous voids and empty spaces within the membranous structure of the SOC. The presence of calcification liquid and exo- and endocytosis are inferred, and the growth of calcium carbonate could be shown using time series samples. However, they have not documented the processes that lead to the construction of the POS and other organic structures during chamber formation.

Undoubtedly, the organic scaffolding built prior to chamber formation is an important factor shaping the characteristic morphology of foraminifera, serving as a template for calcification. When the foraminiferal test is dissolved, the organic structure is revealed and it has the same overall morphology as the calcareous part (Banner and Williams, 1973). Despite the suggestion that pseudopodial activity plays a key role in this process, little is known about the mechanism. Spindler and Röttger (1973) first stated that it is pseudopodia that secrete the organic layer using optical microscopy, working with Heterostegina depressa. However, due to the 
low resolution of optical microscopy, they were unable to see the details of the process and this provided no solid evidence other than speculation.

Although foraminifera are widely used for palaeoenvironment modelling, a total understanding of the foraminiferal calcification process is still lacking, impacting the accuracy of predictions made from foraminifera-based data. An accurate overview and model of the chamber formation by pseudopodia and the calcification process in calcareous foraminifera is therefore urgently needed to better our understanding of paleoceanographic proxies. To fill this knowledge gap, this study aims to elucidate the role of pseudopodial activities on the formation process of the foraminiferal chamber and its organic structures within the calcareous wall using the benthic foraminifera Ammonia beccarii, which has been used in a few relevant previous studies (e.g. Toyofuku et al., 2017), as a model system. We combined differential interference contrast (DIC) microscopy and scanning electron microscopy, capturing DIC images through a time lapse to document the pseudopodial activities during chamber growth, and we carried out SEM observations for specimens fixed at different time slices during the chamber formation process in order to visualize organic structures at the sub-micron order.

\section{Materials and methods}

\subsection{Sample collection and laboratory culture}

Living foraminifera were collected from brackish-water salt marsh sediments of Hiragata Bay, Natsushima-cho Yokosuka, Japan $\left(35^{\circ} 19^{\prime} 21^{\prime \prime} \mathrm{N}, 139^{\circ} 38^{\prime} 5^{\prime \prime} \mathrm{E}\right)$, in the spring of 2015. Surface (top $5 \mathrm{~mm}$ ) sediments were collected and transported to the laboratory to serve as a stock from which individuals of the benthic calcareous foraminifera Ammonia beccarii (sensu De Nooijer et al., 2009) were isolated. Living specimens were recognized by their bright yellow colour and visible pseudopodial activity. They were cleaned from excess sediment and debris under a stereo microscope (SteREO Discovery.V12, Zeiss Co. Ltd.), transferred to filtered $(0.2 \mu \mathrm{m})$ natural seawater (salinity ca. 35) and placed in a petri dish. The petri dishes were maintained at $20^{\circ} \mathrm{C}$ and, twice a week, a small amount of live microalgae (Dunaliella tertiolecta, NIES-2258) were added. Within a few days of feeding, some individuals started chamber formation and were selected for observation.

\subsection{Optical observation settings of chamber formation}

Chambers in the process of formation were observed using an inverted DIC microscope (Axio Observer Z1, Zeiss, Germany). Time-lapse images were captured automatically by the digital microscope software Axiovision (Version 4.6). Time intervals between shots varied from $10 \mathrm{~s}$ to $10 \mathrm{~min}$, but typically the interval was $1 \mathrm{~min}$. Magnifications of the available objective lenses were $\times 10, \times 20 \times 40$, and $\times 63$. A heat cut filter was applied to reduce damage on the living individuals inflicted by the image capture process.

\subsection{Microstructure observation and EDS analysis}

All specimens were fixed simultaneously using a fixing solution $(3 \%$ paraformaldehyde, $0.3 \%$ glutaraldehyde, $2 \% \mathrm{NaCl}$ in PBS buffer, $\mathrm{pH} 7.8$ ) and subsequently stored in $2.5 \%$ glutaraldehyde at $4{ }^{\circ} \mathrm{C}$ to avoid any morphological changes in the cell material through dehydration. They were then washed in $0.2 \mu \mathrm{m}$ filtered seawater and post-fixed with $2 \%$ osmium tetraoxide filtered seawater solution for $2 \mathrm{~h}$ at $4{ }^{\circ} \mathrm{C}$. Following that the specimens were rinsed with distilled water and conductive staining was performed by incubating in $0.2 \%$ aqueous tannic acid (pH 6.8) for 30 min (Willingham and Rutherford, 1984). After another wash with distilled water, specimens were further treated with $1 \%$ aqueous osmium tetraoxide for $1 \mathrm{~h}$. Finally, they were dehydrated in a graded ethanol series and critical point dried (JCPD5; JEOL Ltd., Tokyo, Japan). SEM observations were carried out on a JSM6700F field emission scanning electron microscope (FE-SEM) in Japan Agency for Marine-Earth Science and Technology (JAMSTEC), Yokosuka, Japan. The elemental composition of all specimens was analysed using a JED 2300 (JEOL) dispersive spectrometer (EDS) equipped on the same JSM6700F FE-SEM at JAMSTEC.

Selected specimens processed for SEM observation were embedded in epoxy resin for the purpose of measuring the elemental composition of the newly forming chamber wall. The epoxy resin fully filled the chamber cavities and was polished to expose the chamber wall being formed, and the exposed surface was coated with a ca. $3 \mathrm{~nm}$ thick osmium foil. After rinsing with distilled water, this polished block was sectioned using an automicrotome to generate relief-free sections of foraminiferal tests, revealing fresh calcite surfaces of chamber walls.

\section{Results}

\subsection{Time series observation with optical microscopy}

We were able to observe the chamber formation process of A. beccarii with DIC 59 times in total. Depending on the size of the chamber, it took about 5-8h to complete the whole process (Table 1). Prior to the start of chamber formation, exceptional activities were exhibited by the expanded pseudopodia. Usually for the purpose of feeding and moving, pseudopodia randomly branches at irregular intervals to arbitrary directions with variable lengths. During the chamber formation process, however, the pseudopodial activity significantly differed. A fan-shaped complex pseudopodial network was constructed (Fig. 1a), expanding from the aperture of the last chamber. This pseudopodial network is arranged in a dense, radiating spray resembling that of a dandelion flowerhead. This unique morphology allowed us to recog- 
Table 1. List of observations using inverted differential interference contrast microscopy. ND stands for "no data".

\begin{tabular}{|c|c|c|c|c|c|}
\hline $\begin{array}{l}\text { Date } \\
\text { (YYYYMMDD) }\end{array}$ & $\begin{array}{r}\text { Length of forming } \\
\text { chamber }(\mu \mathrm{m})\end{array}$ & $\begin{array}{r}\text { Width of forming } \\
\text { chamber }(\mu \mathrm{m})\end{array}$ & $\begin{array}{r}\text { Test diameter after } \\
\text { observation time (min) }\end{array}$ & $\begin{array}{r}\text { Recorded stages of } \\
\text { chamber formation }(\mu \mathrm{m})\end{array}$ & Chamber formation \\
\hline 20140401 & 108.12 & 42.24 & 300 & 248.05 & Middle-last \\
\hline 20140402 & 151.45 & 56.31 & 60 & 282.63 & Initial stage only \\
\hline 20140404 & 61.34 & 50.22 & 270 & 135.68 & Middle stage-last \\
\hline 20140407 & 100.58 & 50.4 & 400 & 210.23 & Initial stage-last \\
\hline 20140408 & 104.26 & 58.3 & 395 & 218.69 & Middle stage-last \\
\hline 20140416 & 119.28 & 61.61 & 385 & 287.01 & Middle stage-last \\
\hline 20140417 & 84.61 & 48.45 & 385 & 163.12 & Middle stage-last \\
\hline 20140422 & 127.53 & 83.74 & 465 & 318.79 & Middle stage-last \\
\hline 20140430 & 88.42 & 56.5 & ND & ND & Initial stage only \\
\hline 20140430 & 88.42 & 56.5 & 345 & ND & Middle stage-last \\
\hline 20140509 & 165.95 & 65.63 & 342 & ND & Middle stage-last \\
\hline 20140522 & 94.98 & 57.48 & 360 & ND & Initial stage-last \\
\hline 20140523 & 82.71 & 65.64 & 380 & ND & Initial stage-last \\
\hline 20140711 & 72.21 & 48.94 & 138 & 141.63 & Late stage-last \\
\hline 20140718 & 112.04 & 62.59 & 336 & 284.88 & Initial stage-last \\
\hline 20140808 & 70.56 & 45.53 & 303 & ND & Initial stage-last \\
\hline 20140822 & 96 & 59.13 & 315 & ND & Initial stage-last \\
\hline 20150410 & 99.89 & 45.64 & 303 & 209.47 & Initial stage-last \\
\hline 20150421 & 96.39 & 28.91 & 396 & 178.38 & Initial stage-last \\
\hline 20150817 & 109.14 & 53.03 & 390 & 234.97 & Initial stage-last \\
\hline 20150820 & 146.44 & 94.87 & 475 & 539.68 & Middle stage-last \\
\hline 20150821 & 188.2 & 102.16 & 405 & 517.24 & Middle stage-last \\
\hline 20150903 & 95.77 & 45.81 & 295 & 266.01 & Middle stage-last \\
\hline 20150904 & 96.8 & 58.19 & 360 & 284.16 & Middle stage-last \\
\hline 20150911 & 103.06 & 41.26 & 125 & 291.85 & Late stage-last \\
\hline 20150917 & 92.81 & 55.55 & 325 & 199.63 & Middle stage-last \\
\hline 20150924 & 93.61 & 51.28 & 345 & 204.19 & Middle stage-last \\
\hline 20151001 & 92.08 & 47.51 & 345 & 312.03 & Middle stage-last \\
\hline 20151014 & 79.14 & 58.7 & 380 & 193.5 & Middle stage-last \\
\hline 20151015 & 81.64 & 55.47 & 370 & 175.45 & Middle stage-last \\
\hline 20151111 & 142.81 & 98.42 & 420 & 361.39 & Middle stage-last \\
\hline 20151118 & 84.96 & 54.82 & 270 & 210.6 & Late stage-last \\
\hline 20151119 & 88.66 & 53.6 & 310 & 218.69 & Middle stage-last \\
\hline 20151120 & 186.12 & 118.27 & 550 & 448.84 & Middle stage-last \\
\hline 20151202 & 102.52 & 57.23 & 380 & ND & Middle stage-last \\
\hline 20160127 & 110.04 & 67.85 & 355 & 258 & Middle stage-last \\
\hline 20160302 & 110.47 & 62.45 & 20 & ND & Late stage-last \\
\hline 20160610 & 121.37 & 39.38 & 325 & 317.71 & Middle stage-last \\
\hline 20160611 & 99.89 & 57.42 & 420 & 206.43 & Middle stage-last \\
\hline 20160612 & 107.65 & 27.7 & 335 & 253.74 & Middle stage-last \\
\hline 20160613 & 132.45 & 61.4 & 140 & 317.63 & Late stage-last \\
\hline 20160614-1 & 102.86 & 54.75 & 325 & 245.19 & Middle stage-last \\
\hline 20160614-2 & 99.83 & 41.69 & 150 & 284.98 & Late stage-last \\
\hline 20160618 & 132.4 & 52.5 & 330 & 288.37 & Middle stage-last \\
\hline 20160619 & 138.03 & 34.84 & 270 & 238.39 & Late stage-last \\
\hline 20160621 & 122.66 & 71.06 & 160 & 277.31 & Late stage-last \\
\hline 20160622 & 101.2 & 40.52 & 465 & 240.3 & Middle stage-last \\
\hline 20160623 & 100.34 & 44.88 & 105 & 259.44 & Late stage-last \\
\hline 20160624 & 84.8 & 50.3 & 60 & 233.67 & Late stage-last \\
\hline 20160929 & 114.94 & 62.78 & 214 & ND & Late stage-last \\
\hline 20170221 & 119.67 & 77.47 & 57 & ND & Late stage-last \\
\hline 20170310 & 106.65 & 65.62 & 21 & 277.45 & Late stage-last \\
\hline 20171201 & 83.14 & 45.8 & 459 & 199.71 & Middle stage-last \\
\hline 20171202 & 151.85 & 97.62 & 50 & ND & Late stage-last \\
\hline 20171206 & 120.69 & 61.47 & 582 & 287.28 & Middle stage-last \\
\hline 20171207 & 77.9 & 55.65 & 248 & ND & Initial stage-last \\
\hline 20180104 & 90.5 & 38.1 & 420 & ND & Middle stage-last \\
\hline 20180105 & 114.3 & 54.3 & 377 & ND & Middle stage-last \\
\hline
\end{tabular}



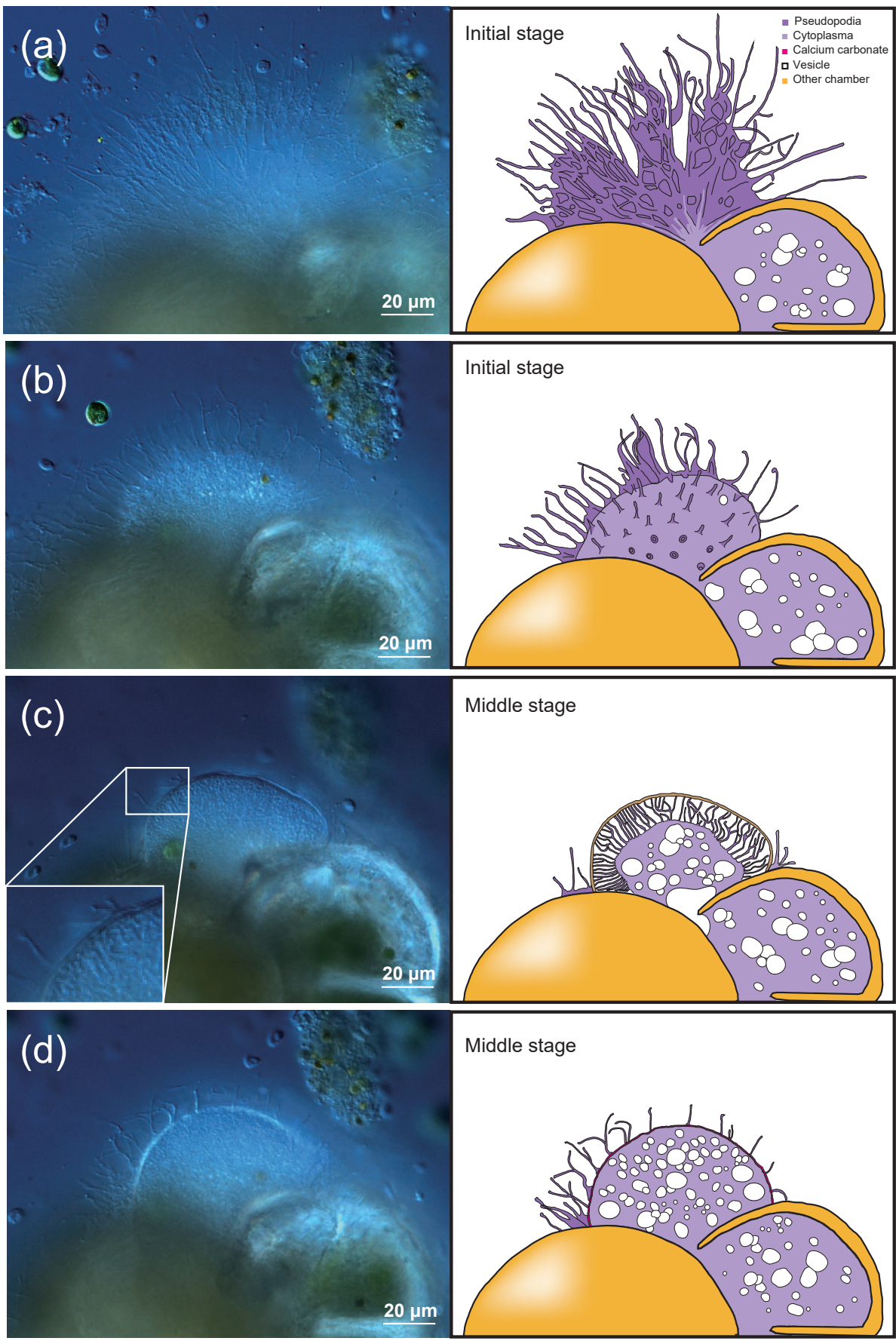

Figure 1. Time series observation of chamber formation by optical microscopy, as seen in the individual observed on 7 December 2017 (see Table 1). The initial stage of chamber formation, where the organic framework is built, is depicted by (a-b). (a) Beginning of chamber formation, defined as $0 \mathrm{~min}$ from the start, indicated by a dense radiating spray of the pseudopodial network. (b) 9 min, when an aggregation of cytoplasm becomes visible around the aperture of the last existing chamber. As this cytoplasm expands, the pseudopodial network starts to retract to the surface of the new chamber to complete the framework. The middle stage, where the organic framework is being prepared for calcium carbonate precipitation, which begins at near the end of this stage, takes place between 15 to 60 min, as depicted by (c-d). (c) $27 \mathrm{~min}$, cytoplasm concentrates and outline of newly forming chamber wall is now clearly visible, pseudopodia still just visible on the surface. (d) $41 \mathrm{~min}$, pseudopodial retracts inside the forming chamber wall. Left: optical microscopy image. Right: the same image with schematic overlay. Colour legend: deep purple = pseudopodia; light purple = cytoplasm; magenta = calcium carbonate in the newly forming chamber; yellow $=$ previously formed chambers. 
nize individuals in the beginning of chamber formation and start our time-lapse observation (from $0 \mathrm{~min}$ ). For an average individual, the events of chamber formation can be sequentially divided into three steps, outlined as follows in a typical time sequence (see Section "Video Supplement").

The initial stage of chamber formation was from 0 to approximately $15 \mathrm{~min}$, although this varied considerably depending on the individual and the size of the newly forming chamber observed, as well as on all stages (Table 1). The initial stage is the stage where the organic framework for chamber formation is built. Following the pseudopodial network construction which takes place from $0 \mathrm{~min}$, an aggregation of cytoplasm (Fig. 1b) quickly became visible around the aperture of the last existing calcified chamber (Fig. 1b). As the cytoplasm expands, the pseudopodial network retracts to the surface of the newly forming chamber. We consider the completion of the organic framework to be the end of the initial stage.

The middle stage of chamber formation took place approximately at around 15-60 min (Fig. 1c-d). During this stage, the foraminifera prepare the organic scaffolding for calcium carbonate precipitation, which begins during this stage. By about $30 \mathrm{~min}$, the cytoplasmic aggregation concentrates in the same shape of a newly forming chamber, like a hemisphere (Fig. 1c). At this point, fine and short pseudopodia have retracted to a certain extent but are still seen on the surface of the structure. A bright band, probably representing calcium carbonate starting to become formed, can be seen on the surface of this. This proceeds to become the chamber wall. Finally, the pseudopodia retracts inside the forming chamber wall (Fig. 1d).

The late stage of chamber formation is defined as the stage where material, inferred to be calcium carbonate, is precipitated extensively to thicken the chamber wall in the newly forming chamber, and took place between around 60 and $400 \mathrm{~min}$ (total time varied among individuals; Table 1). We define the start of the late stage as when the pseudopodia begins to expand again to cover the organic scaffolding, and also when the whole organic scaffolding is covered by a layer of calcium carbonate (with pores becoming visible under light microscopy). At the start of this stage, the pseudopodia expand again to form a dense network, this time in thicker strands (Fig. 2a). The length of all pseudopodia appear to be remarkably regular. Calcium carbonate continues to be precipitated in the forming wall. At this point, the overall outline of the newly forming chamber is basically fixed. Pseudopodial movement can be seen inside the forming chamber (Fig. 2a). Cytoplasm aggregate that filled the newly forming chamber retreats to the previously formed chamber, leaving an empty space in the new chamber. After that, a network of pseudopodia is present in the forming chamber, the chamber wall of which thickens (overall distance between OOL and POS increased over time) and the pores become increasingly and clearly visible (from Fig. 2b-c; also see video in Section "Video supplement"). Chamber thickening contin- ues to occur from this point onwards, generally at around 120-400 min (Fig. 2c). During this process, the density of the pseudopodial network on the chamber wall surface increased and wraps the chamber wall like a mesh. As the chamber wall thickening completes (at approximately $400 \mathrm{~min}$, this is actually quite variable; the specimen shown in Figs. 1-2 had completed this by $248 \mathrm{~min}$ ), the mesh-like pseudopodial network on the surface disappears (Fig. 2d). We consider this to indicate the termination of chamber formation process. After this, the individual starts to show the usual type of pseudopodia movement (typical of reticulopodia).

\subsection{Ultra-micro observations on the forming chamber wall}

The process of chamber formation is classified into three stages, as outlined above. Specimens exemplary of each stage were observed with a scanning electron microscope. Figures 3-6 show microstructures at different stages of chamber formation seen by the SEM.

\subsubsection{Initial stage}

In the initial stage of chamber formation, the test was entirely covered with pseudopodia and organic layer-like structures (Fig. 3a); some parts of these covering structures were peeled off during the sample preparation process. Focusing on the chamber being formed, it was possible to observe the OOL and the POS (Fig. 3b), with the POS being visible from gaps in the OOL. The interspace between the two layers was narrow (Figs. 3b-c). Even at high magnification, the outer surface of the OOL itself is a relatively smooth layer-like structure (OOL in Fig. 3b-c). In some cases, the pseudopodia can be seen directly expanding from the OOL (Fig. 3c). The primary organic sheet (POS) can also be observed (Fig. 3b) and is relatively robust, covered by numerous protrusions. These are convex, frustoconical structures about $1 \mu \mathrm{m}$ in width (Fig. 3a-b) and represent pore plates which corresponds to pores. These were simultaneously formed when the POS was constructed (green coloured in Fig. 3c-d). Projections $(<\mu \mathrm{m})$ were observed on the cytoplasmic surface of OOL (light green in Fig. 3b). Spherical structures can be seen on the OOL (blue coloured in Fig. 3C; some appeared crushed, probably due to the critical point drying process), and similar structures could also be found on the POS (blue coloured in Fig. 3d and e). The size of these spherical structures varied from 50 to $500 \mathrm{~nm}$. On the $\mathrm{OOL}$, some elongated structures, inferred to be pseudopodia, appeared to have a form like that of a sausage chain (Fig. 3b); the diameter and interval of contractions were variable. The bulging part contained only cavities, and this form might be associated with peristalsis. It is known that pseudopodia transport mitochondria and vesicles (Travis and Bowser, 1991; Cedhagen and Frimanson, 2002), and it is possible that this peristaltic structure has important roles in such transportation. 

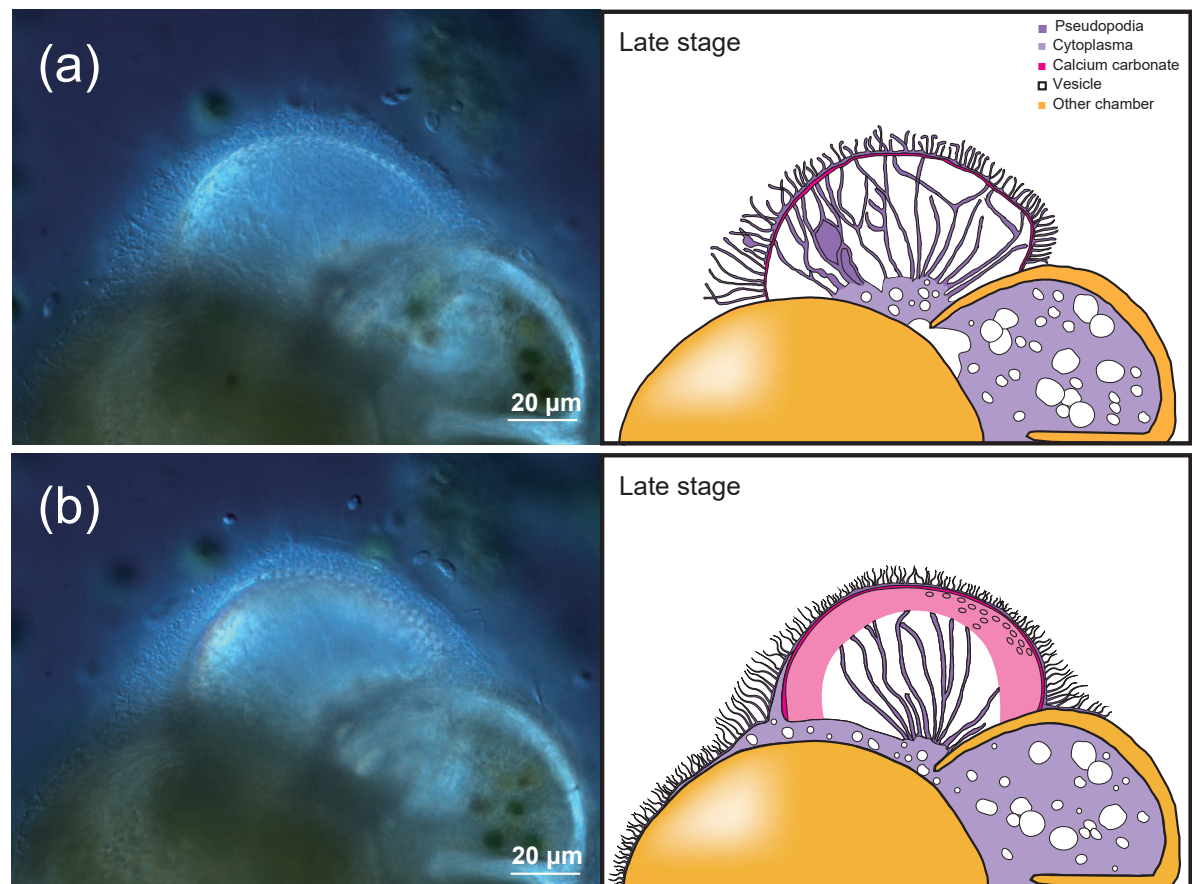

Late stage
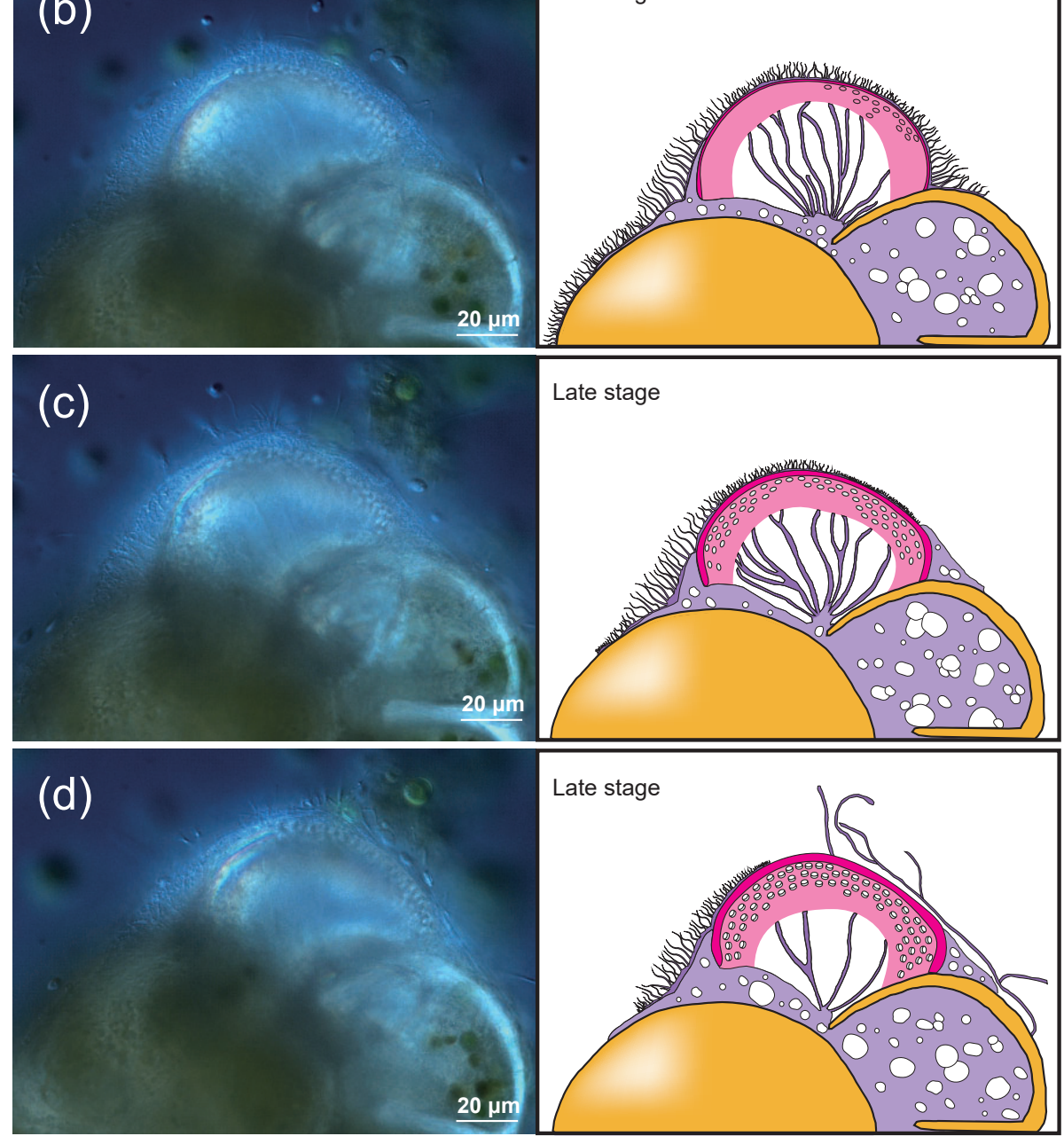

Figure 2. Time series observation of chamber formation by optical microscopy (continued), as seen in the individual observed on 7 December 2017 (see Table 1). The late stage of chamber formation, where calcium carbonate is extensively precipitated and the chamber wall is thickened, taking place from around $60 \mathrm{~min}$ after the start of chamber formation (total time varies considerably among individuals). (a) 65 min, pseudopodia expands again to form a dense network but in thicker strands than seen in previous stages. (b) 100 min, a network of pseudopodia is seen in the new chamber, the chamber wall of which thickens. (c) 124 min, chamber wall thickening continues. (d) 180 min, chamber wall thickening is nearly completed and the pseudopodial network begins to disappear, indicating that the end of the chamber formation process is near (actual completion was at $248 \mathrm{~min}$ for this individual). Left: optical microscopy image. Right: the same image with schematic overlay. Colour legend: deep purple = pseudopodia; light purple = cytoplasm; magenta $=$ calcium carbonate in the newly forming chamber; yellow $=$ previously formed chambers. 


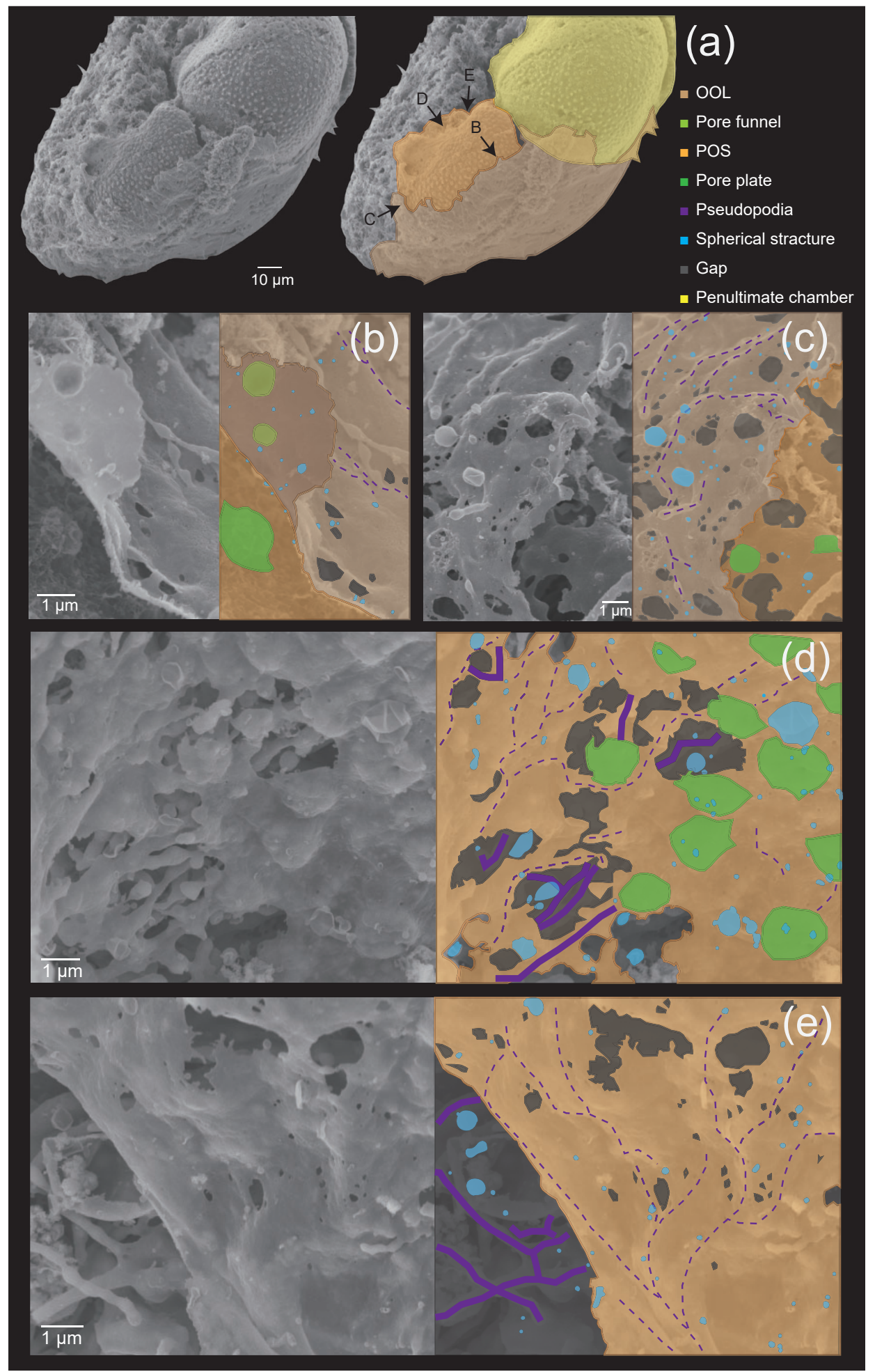

Figure 3. Microstructures during the initial stage of the chamber formation, shown by SEM images on the left, supplemented by schematic explanation on the right. (a) Overview of a specimen showing the OOL covering both the newly forming and older chambers. (b-c) Magnified images showing the OOL and the POS. (d-e) Magnified image of the POS construction front showing the weaving action of pseudopodia. (e) The same POS construction front showing the membranous pseudopodia extending so as to close a large hole (white arrow). Colour legend: brown = OOL; orange $=$ POS; purple = pseudopodia/cytoplasm; light green = pore funnel on the OOL; green = pore plate; blue $=$ spherical structures; gray $=$ gap. Thick lines indicate membranous pseudopodia and dotted lines indicate framework pseudopodia. 
No crystals were found between the POS and the OOL, indicating that no calcium carbonate has been deposited at this stage, supported by the fact that the SEM-EDS analyses showed an absence of calcium signals (Fig. 6a).

\subsubsection{Middle stage}

At the middle stage, the interspaces among the framework structure constructed by the pseudopodia has been filled to a much larger extent than in the initial stage, with much fewer gaps (about 5-200 nm; grey coloured in Fig. 4b and c) that could be seen. Nevertheless, calcium carbonate precipitation has already started between organic layers in some parts of the forming chamber (Fig. 4d). Upon closer observation, these were revealed to consist of needle-like structures that covered the surface of the POS, close to the previously formed chamber. These needle-like structures were confirmed to be crystals of calcium carbonate precipitating vertically between the OOL and the POS by EDS observation (Fig. 6b). Therefore, the precipitation does not start at the same time across the entire chamber, but instead begins locally right after the completion of organic layer construction. At this point, there are still small gaps between independent crystals. It can also be noted that the framework structure formed by pseudopodia appears to have a certain directionality in growth.

Numerous, rather regularly spaced pores (about $1 \mu \mathrm{m}$ ) can be clearly observed on the crystalline layer (Fig. 4a). In the part where OOL was curled up to reveal the inner side (see Fig. 3b), convex structures corresponding to pore lining were seen (Fig. 4d). This has been termed "pore funnel" by Hottinger (2006), a term that we adopt here. Interestingly, pores cannot be seen at this stage from the outer side on the OOL with SEM observation (Fig. 4a-b), and the OOL appears to be entirely smooth in the parts where the framework has been filled completely. We interpret this as being due to a layer of cytoplasmic material that also fills the pore lining (i.e. the "well") during chamber formation, which regresses after the completion of chamber formation (and therefore becomes visible under SEM). As discussed previously, however, pores can still be seen during chamber formation using light microscopy due to the semi-transparent nature of the organic layers as well as the thin calcium carbonate layer. Algal cysts including Dunaliella individuals can be seen overlaying the OOL (Fig. 4a). The OOL is a continuous structure that envelopes the entire test, and it extends to the newly forming chamber from the aperture of the previously formed chamber. In some parts where calcium carbonate precipitation has not yet taken place, the outer surface of the POS can be seen (Fig. 4a and c) and like in the initial stage, many frustoconical structures about $1 \mu \mathrm{m}$ in width are seen (Fig. 4c). Spherical structures (blue coloured in Fig. 4b-c), about $50-500 \mathrm{~nm}$ in size, could be seen on both the OOL and the POS as in the initial stage.

\subsubsection{Late stage}

In this final stage (Fig. 5), the construction of organic layers has been fully completed, and a layer of calcium carbonate began precipitation across the entire newly forming chamber. The OOL is therefore seen as uniformly smooth and without gaps, from the outer side (Fig. 5b). A cross section through the forming chamber wall at the late stage clearly shows three completed layers (corresponding to the IOL, the POS, and the OOL, respectively, from the inner layer outwards, in that order) and two layers of precipitating calcium carbonate sandwiched between the IOL and the POS as well as between the POS and the OOL (Fig. 5c). EDS analyses obtaining signals of $\mathrm{Ca}, \mathrm{C}$, and $\mathrm{O}$ simultaneously (Fig. 6c) clearly indicated high $\mathrm{Ca}$ signal distribution being detected these two layers, showing that these layers are calcium carbonate in nature.

The precipitation of calcium carbonate crystals, continuing from the middle stage, leads to carbonate crystals becoming increasingly densely packed, with gaps between crystals completely disappearing by the end of the late stage (which marks the end of chamber formation). In the figured specimen observed in Fig. 4, the thickness of the calcium carbonate layer is about $1 \mu \mathrm{m}$ between the OOL and the POS, and about $0.3 \mu \mathrm{m}$ between the POS and the IOL.

As in the middle stage, the exterior of the OOL appears to be smooth (i.e. pores cannot be seen yet) (Fig. 4d). The IOL, however, when seen from the cytoplasm side, is seen to be covered by regular depressions that correspond to the convex side of the pore plate on the POS (which may be named the "inner pore") (Fig. 5d). The IOL can therefore be considered to have the same shape as the POS. Spherical structures could also be observed in the late stage on the surface of the OOL, but the size of these structures was more variable than in the earlier stages, ranging from $50 \mathrm{~nm}$ to $1 \mu \mathrm{m}$ (Fig. $4 \mathrm{c}-\mathrm{d}$ ). Furthermore, similar structures could also be observed on the IOL (not shown).

\section{Discussion}

\subsection{The weaving of organic layers during chamber formation}

This study is the first to observe the detailed making of organic layers during chamber formation and revealed that the layers are actually woven by pseudopodial activity. A schematic diagram is presented in Fig. 7, which outlines the general observations. The basis of organic layer formation is the interweaving of a pseudopodial framework (Fig. 7a), the interspaces of which is then filled in with a further layer of pseudopodial material, resulting in a complete organic layer. The pseudopodia are observed to form a dense framework (purple dotted lines in Figs. 2a, 3 and 7), which is then overlaid by a layer of membranous pseudopodia which fills the in- 


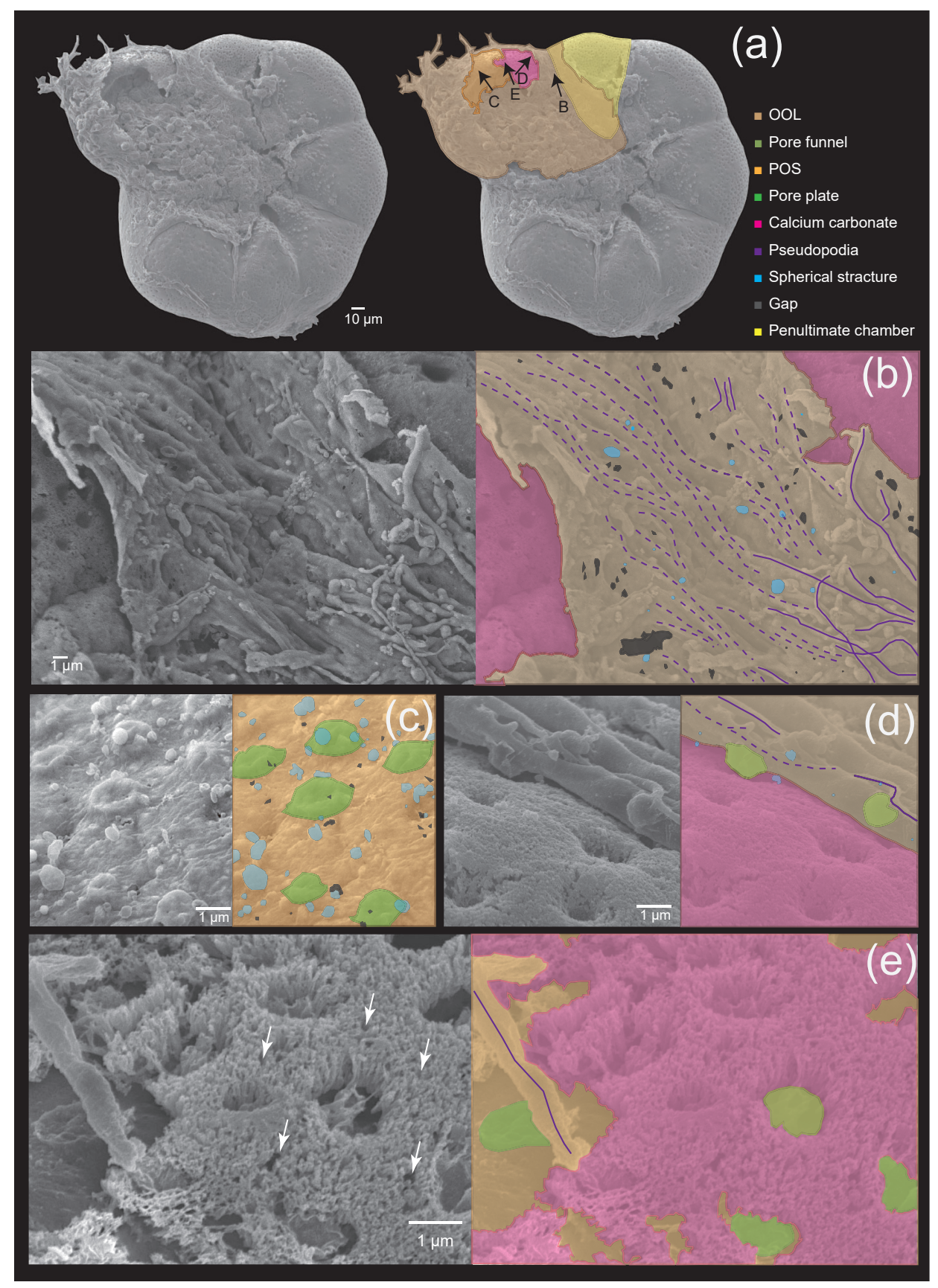

Figure 4. Microstructures during the middle stage of the chamber formation shown by SEM images on the left, supplemented by schematic explanation on the right. (a) Overview of the ventral side of a specimen, showing the cytoplasm covering the newly forming chamber. (b) Magnified image showing the OOL on the suture, between the new chamber and the previous chamber. (c) A higher magnification image of the POS showing spherical structures on the POS. (d) Image showing the matching relationship between convex structures on the cytoplasmic surface of the OOL and the pore. (e) Needle-like crystals of calcium carbonate between the IOL and the OOL, with white arrowheads indicating clear gaps between the crystals. Colour legend: brown =OOL; orange $=$ POS; purple $=$ pseudopodia/cytoplasm; light green $=$ pore funnel on the OOL; green = pore plate; magenta = calcium carbonate; blue = spherical structures; gray = gap. Thick lines indicate membranous pseudopodia and dotted lines indicate framework pseudopodia.

terspaces (Fig. 3e). In the OOL, numerous spaces of $100 \mathrm{~nm}-$ $1 \mu \mathrm{m}$ can be seen (gray in Figs. 3, 5, and 7), which represent the interspaces between the framework that is yet to be filled. In some instances, the membranous pseudopodia were observed during the process of filling the interspaces, sometimes from more than one direction (e.g. Fig. 3e), by a grad- 


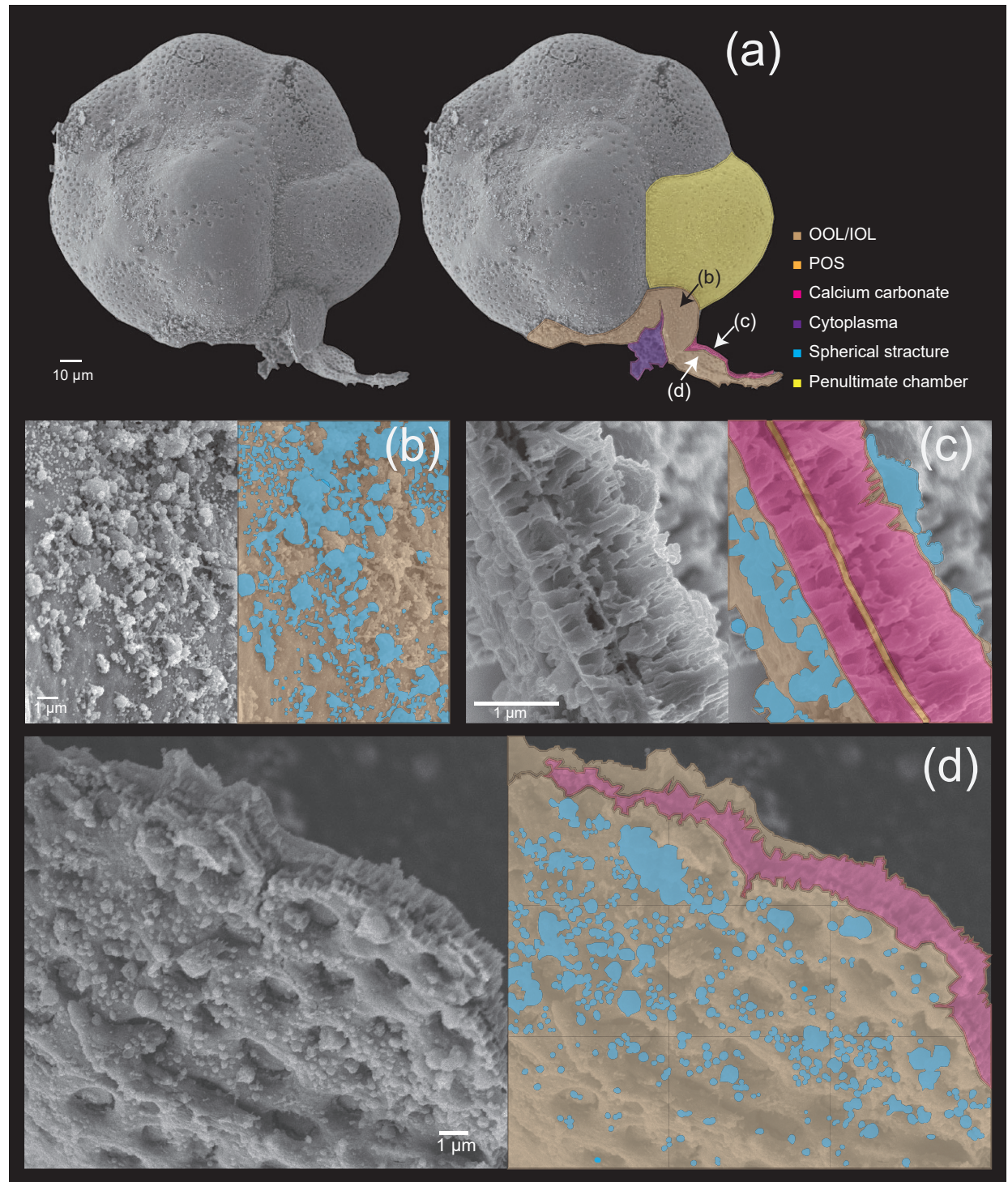

Figure 5. Microstructures during the late stage of the chamber formation shown by SEM images on the left, supplemented by schematic explanation on the right. (a) Overview of the dorsal side of a specimen, with the newly forming chamber on the bottom. (b) Magnified image of the OOL seen from the outside. (c) Image showing a cross section through the forming chamber wall. (d) A magnification of the IOL seen from the inner side, showing pores and lots of spherical structures. Colour legend: brown $=$ OOL/IOL; orange $=$ POS; purple $=$ pseudopodia/cytoplasm; light green $=$ pore funnel on the OOL; green $=$ pore plate; magenta $=$ calcium carbonate; blue $=$ spherical structures.

ual, webbed expansion. In short, a framework is constructed by a pseudopodial network, which is then overlaid and the interspaces filled in by a layer of membranous pseudopodia. According to a recent study (Nagai et al., 2018), the three organic sheets (OOL, POS and IOL) initially appear to be independent even at the very early stage of chamber formation, when the total thickness of the whole organic sheet being less than $1 \mu \mathrm{m}$. We expect that these organic sheets themselves are ultimately expanded from a single root, but separate branches of pseudopodia form each organic sheet.
The importance of organic layers in the early stages of chamber formation has been speculated in previous studies, but little was known about its origin. It was previously thought that the organic layer was secreted from the pseudopodia (e.g. Angell, 1967; Röttger, 1974; Hemleben et al., 1986), and Spindler and Röttger (1973) reported that the organic layer seems to be connected with pseudopodia. These studies were largely limited in that their magnification (only light microscopy was available then) was not sufficient in resolution to observe the detailed process. The process documented herein provides evidence for an entirely novel model 


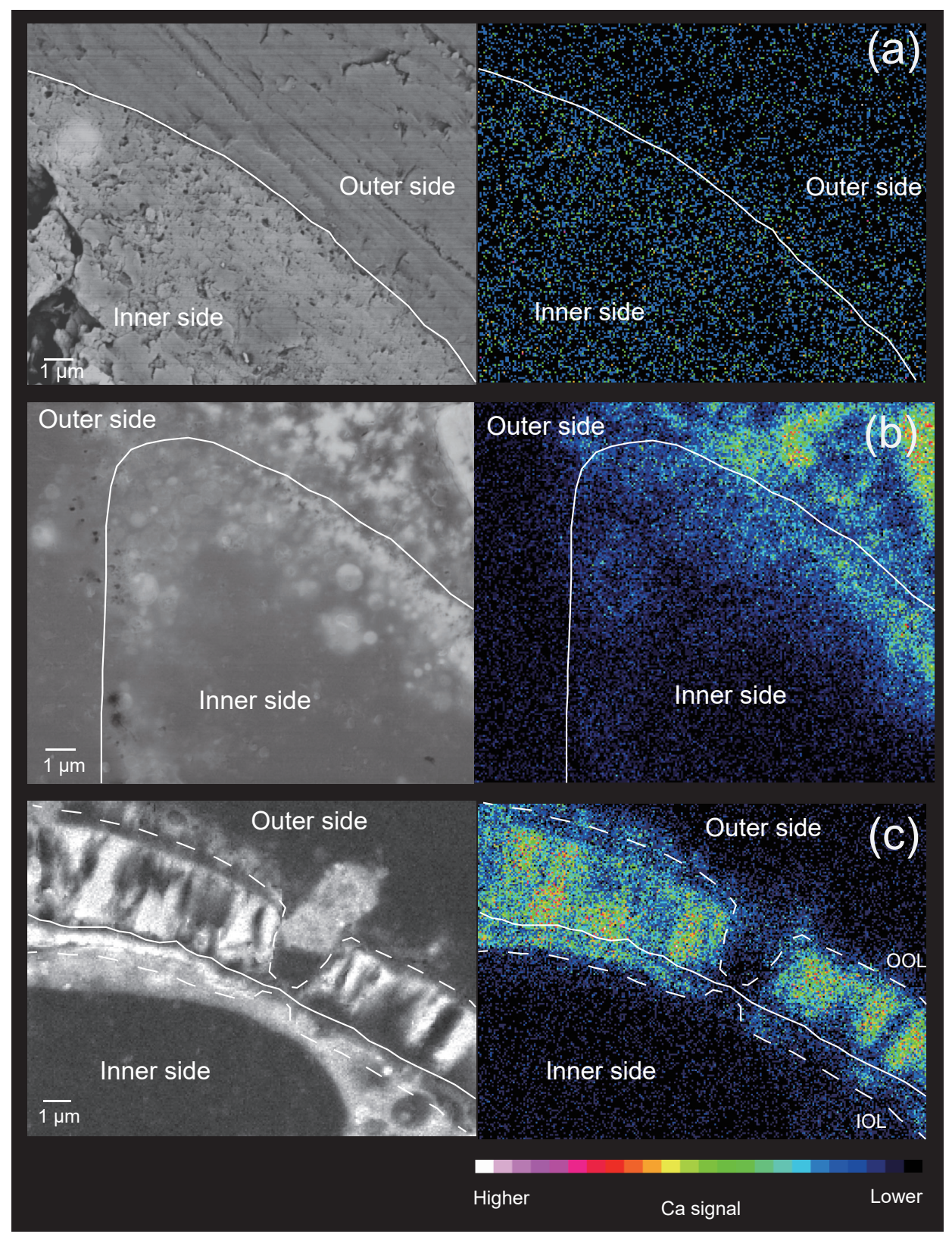

Figure 6. Elemental maps of cross sections through the forming chamber wall at different stages, shown by SEM-EDS analyses. (a) Initial stage. (b) Middle stage. (c) Late stage. White lines indicate the position of the POS. The false colour maps indicate the intensity of calcium signals, corresponding to the legend shown on the bottom. Thick lines indicate membranous pseudopodia and dotted lines indicate framework pseudopodia.

in that the pseudopodia themselves weave the organic layers (Fig. 3c-e, Section "Video Supplement") - in other words the organic layer is part of the cytoplasm.

The reason why the space between IOL and POS is narrower than between OOL and POS (meaning the inner calcareous layer is thinner than the outer) is presumably caused by the difference in the growth rate of calcareous material between the inner side and the outer side. Assuming that the materials for chamber formation are transported from the seawater, it can be presumed that the inner side will become thinner because the chamber wall is formed and material transportation is more restricted on the inner side.

\subsection{Pore formation}

Fine-scale observations from the present study allowed us to reconstruct the actual steps in pore formation. As shown already in previous studies (Bé et al., 1979; Spero, 1988), the structure known as a "pore" in foraminifera is actually a com- 

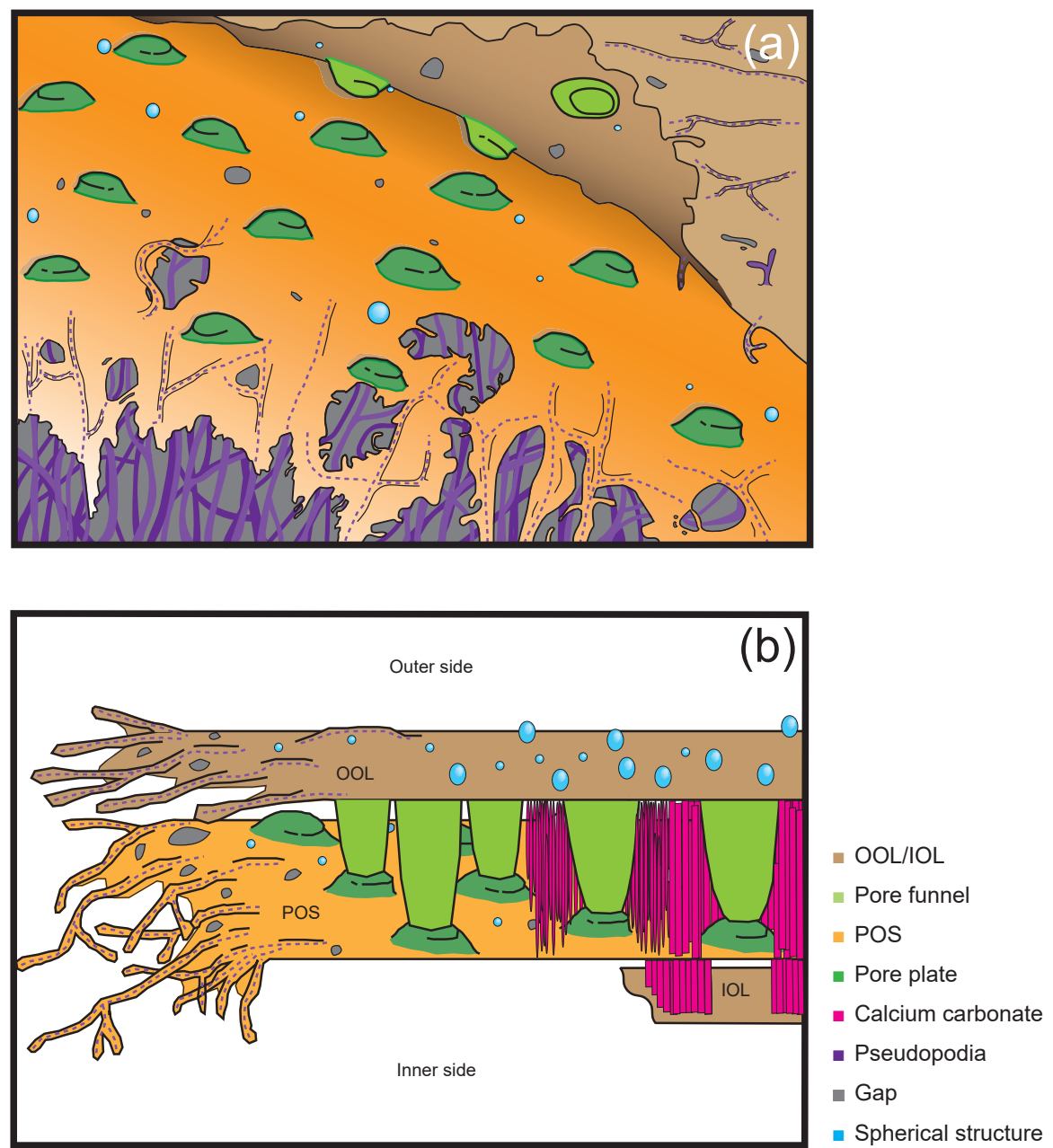

Figure 7. Schematic illustrations of chamber formation. (a) Construction of organic layers by pseudopodial weaving and subsequent gapfilling. (b) The entire chamber formation process from the initial stage on the left side to the late stage on the right side. Colour legend: brown $=\mathrm{OOL} / \mathrm{IOL}$; orange $=\mathrm{POS}$, purple = pseudopodia/cytoplasm; light green $=$ pore funnel on the OOL; green $=$ pore plate; magenta $=$ calcium carbonate $($ see Fig. $5 \mathrm{e}$ ); blue = spherical structures; gray = gap.

posite structure formed by two opposing wells converging at the POS, one opening towards the outer side located on the OOL and one opening towards the cytoplasm side located on the POS (and same on the IOL). The POS-IOL well has been called the pore plate in previous studies (e.g. Haynes, 1981). These pore plates can also be seen on the organic layer template when fossil foraminiferal tests are dissolved (Bannar et al., 1973; Banner and Williams, 1973; Hottinger and Dreher, 1974; Cadre et al., 2003; Ní Fhlaithearta et al., 2013). Pore plates seem to be dented on the IOL side, according to a previous study (see Fig. 3c in Nagai et al., 2018). Therefore, the pores are not actually pass-through structures formed at once but are instead formed in unison by separate processes on the OOL and the IOL. Pores have been suggested to be used for respiration (e.g. Berthold, 1976; Leutenegger and Hansen, 1979). As $\mathrm{O}_{2}$ and $\mathrm{CO}_{2}$ used in respiration are nonpolar molecules, they are able to pass through the cell mem- brane. As such, the existence of pore plates made from cytoplasm seen in the present study should not influence respiration. Our observations show that in the initial stage of chamber formation, the pore plate (visible as frustoconical structures of about $1 \mu \mathrm{m}$ ) is already present when the POS is woven, at the growth front. Pore funnels, about $0.5 \mu \mathrm{m}$ in size, which pair up with the pore plate in the same location (but open to the opposite direction), are formed on the OOL. This structure and the pore plate collectively form the pore, and there is no space between the two for calcium carbonate to precipitate, and therefore the pore is not calcified. Pore plates and pore funnels smoothly peeled off from one another (Figs. $3 b$ and 7a), suggesting that pore plates and pore funnels belong to independent organic sheets formed from separated pseudopodia. Pore plates and funnels were gently adhered to each other before calcification started. It is thought that pore plates and pore funnels are formed simultaneously 
face to face, during the organic sheet formation. All hyaline foraminifera that have been observed in detail possess pores. Since pores are not pass-through and are formed as the framework for the organic layer (i.e. OOL, POS, and IOL) formation is woven, and since the layers are somewhat flexible before calcification, one possible speculative function for pores is to serve as a connective structure between OOL and IOL. In this scenario, the pores "staple" the organic layers of the forming chamber together, so that the sites of calcification maintain a consistent thickness and form throughout the chamber while calcification occurs.

\subsection{Spherical structures}

The existence of spherical structures on the surface of organic layers have been reported in previous studies (Angell, 1967; Spero, 1988), but their function and significance have not been mentioned. A recent study (Nagai et al., 2018) utilized focused ion beam (FIB) technology to process SEM samples in order to visualize calcium carbonate and organic layers on the same semi-thin section. They were able to observe the presence of spherical structures in the site of calcification, and they might be responsible for exo- and endocytosis. The spherical structures increase the surface area and probably serve to improve the material exchange efficiency, by increasing the contact surface area with seawater. In the present study, we could observe numerous spherical structures on all three organic layers, including the OOL, the POS, and the IOL. This indicates that the spherical structures probably play important roles in material exchange during calcification for both the outer and inner calcified layers, and as the spherical structures are inferred to result from the activity of the organic layers this further strengthens the active role of these layers in calcification (i.e. they are not mere templates). The number of spherical structures increased as the chamber formation progressed. There is a variation in the size of spherical structure from ca. $50 \mathrm{~nm}$ to $1 \mu \mathrm{m}$. Small ones were relatively more numerous at the initial stage and large ones were relatively more numerous at the late stage, but all sizes of the spherical structures are found across all stages.

\subsection{Prospects for calcification model}

Until now, the exact process of calcium carbonate precipitation, in terms of how precipitation was related to the degree of isolation of the site of calcification, remained largely unclear (Erez, 2003; De Nooijer et al., 2014). In the present study, the sequence of events during calcification was made clear by time-series observations, and importantly both the formation of the organic layer and calcium carbonate precipitation were observed together. It is significant that during the middle stage, although the overall shape of the forming chamber has already been formed by framework-like pseudopodia, the precipitation of calcium carbonate was seen to initially start before the framework pseudopodia have been fully covered and filled by membranous pseudopodia. The organic layers (especially well-observed in the OOL and the POS) still contained numerous gaps $<1 \mu \mathrm{m}$ in size, which we interpret as maintaining the exchangeability of seawater and elements contained within, for the initial part of calcium carbonate precipitation. The site of calcification is therefore interpreted to be still open to seawater during the middle stage. In the late stage, however, the organic layers have been completely filled by membranous pseudopodia and no such gaps remain. At this stage, therefore, the site of calcification is closed from the surrounding seawater. Hence, we interpret that during the late stage the elements required for calcification must be selectively taken up by biological means such as exo-endocytosis or ion pumps through the OOL. Although we could not observe the IOL in detail during this process (due to its position below the POS), the IOL most likely receives the required elements through pseudopodial transport during the late stage, although whether this originates directly from the forming chamber or the previous chambers cannot be ascertained yet. Previous evidence (e.g. Toyofuku et al., 2008; De Nooijer et al., 2009) appears to suggest that calcium and carbonate are transferred from the cellular material inside the previously formed chamber. The elemental composition of the inner calcified layer, formed between the IOL and the POS, is probably more closed and strongly affected by cellular processes compared to the outer calcified layer between the OOL and the POS. Therefore, the magnesium contents of the inner layer may differ from the outer layer and pure calcite may be precipitated in the inner side. The POS has been widely considered to be the only template for calcification (Hemleben et al., 1986), but recent research has revealed that calcium carbonate precipitation also occurs on the other organic layers (Nagai et al., 2018). It was also shown that the POS gradually becomes obsolete as the chamber matures towards completion of thickening. Therefore, the true role played by the POS during calcification should be reconsidered. A likely function of the POS is that by doubling the surface area on which precipitation occurs, the existence of the POS doubles the rate of chamber formation. Considering that the mobility of foraminifera is highly limited during chamber formation, increasing the efficiency of chamber formation is probably beneficial and adaptive for the foraminifera.

It is well known that the chemical and isotopic compositions of calcareous foraminifera tests differ significantly from those precipitated inorganically, and the compositions also differ among different species. This effect is collectively known as the "vital effect" (Urey et al., 1951) and has been a great hindrance to the use of foraminifera tests as geochemical proxies, for example to reconstruct palaeoclimates. In attempt to explain the vital effect, Nehrke et al. (2013) proposed a transmembrane transfer-passive transfer (TMT/PT) model by observing $\mathrm{Mg} / \mathrm{Ca}$ ratio during calcification, assuming that a low ratio indicates active transport (i.e. transmembrane transfer sensu Nehrke et al., 2013) and a high ra- 
tio indicates passive transport, as $\mathrm{Mg}$ is discriminated against in $\mathrm{Ca}$ channels in active transport. Their observations indicated that passive transportation predominates at the early period of calcification, with active transport becoming dominant at later periods. This is consistent with the results outlined above from our observations during the present study, but we were able to reveal the reasons behind the differences in $\mathrm{Mg} / \mathrm{Ca}$ ratios in early and later periods of calcification, which is that during the middle stage the site of calcification has not yet been fully isolated from the surrounding seawater. This is a key finding with respect to what actually causes the vital effect, in that the construction process of the organic layers can significantly influence when the site of calcification becomes isolated, leading to differences in chemical and isotopic compositions of the test by the proportion of contributions from passive vs. active transport. The elemental analysis of fluid at the site of calcification, however, is still currently unmeasurable due to technical limitations. Nevertheless, because magnesium ions are an inhibitor of calcification, it can be speculated that during biomineralization magnesium ions are actively discriminated and removed from the fluid at the site of calcification (Zeebe and Sanyal, 2002). Therefore, it is presumed that calcite with low $\mathrm{Mg} / \mathrm{Ca}$ precipitates even around the POS. It is reported in many species that the foraminiferal $\mathrm{Mg} / \mathrm{Ca}$ is high around the POS, but it is still much lower than $\mathrm{Mg} / \mathrm{Ca}$ estimated from inorganic precipitation experiments (De Nooijer et al., 2014). The elemental partitioning in foraminiferal tests must be strongly controlled through the elemental composition of the fluid in the SOC, which is a key subject for future studies.

\section{Conclusions}

Calcareous foraminifera are a highly important group in palaeoclimate reconstruction and as indication fossils, by using their chemical and isotopic composition as a geochemical proxy. A major problem was that such compositions differed greatly from inorganic calcium carbonate under the same environment. The key finding of the present study is that one main contributor to this "vital effect" is in fact the proportion of contributions from passive vs. active transport in material transfer shifts during calcification, which is directly linked to how the three major organic layers (i.e. the OOL, the POS, and the IOL) are constructed. For the first time, this study revealed that the organic layers are in fact woven by a framework-like pseudopodia network that is then overlaid by an overlaying layer of membranous pseudopodia, closing the gaps in the framework and thus forming a complete organic layer. We show that calcification has already started when the site of calcification is still able to passively exchange elements (e.g. $\mathrm{Mg}$ ) with seawater; but the majority of wall thickening occurs when it is completely isolated and the only means of element exchange is through active transport. This agrees with and explains the differences in $\mathrm{Mg} / \mathrm{Ca}$ ratios in early and later periods of calcification observed in previous studies (e.g. Nehrke et al., 2013). As such, we resolved a key "missing piece" in understanding foraminiferal calcification that has mystified us for more than a decade. This study exemplifies the importance of extensive rearing and in-depth observations of a living species in order to correctly use biominerals as a geochemical proxy.

Data availability. Original images of data used in Table 1 are available on Figshare, https://doi.org/10.6084/m9.figshare.7269440. All other data supporting the results of this article are included within the article and its Supplement.

Video supplement. The Video Supplement contains a time-lapse observation of the entire chamber formation process of an Ammonia beccarii individual, taken on 7 December 2017. This is the same individual used for Figs. 1-2. The video can be accessed at https://doi.org/10.5446/38290.

\section{Information about the Supplement}

The Supplement contains original high-resolution photographs used in Figs. 1-2.

Supplement. The supplement related to this article is available online at: https://doi.org/10.5194/bg-15-6773-2018-supplement.

Author contributions. Scientific conception and experimental design were carried out by YN and TT. Data acquisition and analysis were carried out by YN, TT and KU. Data processing was carried out by YN. Data interpretation was carried out by YN, TT, CC, and JT. YN, TT, CC, KU, RW and JT wrote and edited the paper. YN and TT contributed equally to this work.

Competing interests. The authors declare that they have no conflict of interest.

Acknowledgements. The authors thank Nanami Kishigami, Sunaho Kubo, Yuki Iwadate, Sachiko Kawada (JAMSTEC), and Shunzo Kondo (JEOL) for their technical assistance and scientific advice on this study. The Axiocam 506 colour camera used for time-series imaging in the present study was kindly lent to us by Carl Zeiss Japan, chiefly for a niconico-JAMSTEC foraminifera live streaming outreach event (http://live.nicovideo.jp/watch/lv309040835, last access: 18 October 2018). This work was supported by a grant from the Faculty of Environment and Information Sciences, Yokohama National University (to Yukiko Nagai), and a JSPS KAKENHI grant, numbers 18H6074 (to Yukiko Nagai) and 25247085 (to Takashi Toyofuku). Jarosław Tyszka received support from the Polish National Science Centre (UMO-2015/19/B/ST10/01944). We thank Inge van Dijk and another anonymous reviewer for constructive comments and suggestions that improved an earlier 
version of this paper.

Edited by: Hiroshi Kitazato

Reviewed by: Inge van Dijk and one anonymous referee

\section{References}

Angell, R. W.: The Test Structure and Composition of the Foraminifer Rosalina floridana, J. Protozool., 14, 299-307, 1967.

Angell, R. W.: Calcification during chamber development in Rosalina floridana, J. Foramin. Res., 9, 341-353, 1979.

Banner, F. T. and Williams, E.: Test structure, organic skeleton and extrathalamous cytoplasm of Ammonia Bruennich, J. Foramin. Res., 3, 49-69, 1973.

Banner, F. T., Sheehan, R., and Williams, E.: The organic skeletons of rotaline foraminifera; a review, J. Foramin. Res., 3, 30-42, 1973.

Bé, A. W. H., Hemleben, C., Anderson, O. R., and Spindler, M.: Chamber formation in planktonic foraminifera, Micropaleontology, 25, 14, https://doi.org/10.2307/1485304, 1979.

Berthold, W.-U.: Ultrastructure and function of wall perforations in Patellina corrugata Williamson, Foraminiferida, J. Foramin. Res., 6, 22-29, 1976.

Cadre, V. L., Debenay, J.-P., and Lesourd, M.: Low ph effects on Ammonia beccarii test deformation: implications for using test deformations as a pollution indicator, J. Foramin. Res., 33, 1-9, 2003.

Cedhagen, T. and Frimanson, H.: Temperature dependence of pseudopodial organelle transport in seven species of foraminifera and its functional consequences, J. Foramin. Res., 32, 434-439, 2002.

De Nooijer, L. J., Toyofuku, T., and Kitazato, H.: Foraminifera promote calcification by elevating their intracellular $\mathrm{pH}$, P. Natl. Acad. Sci. USA, 106, 15374-15378, 2009.

De Nooijer, L. J., Spero, H. J., Erez, J., Bijma, J., and Reichart, G. J.: Biomineralization in perforate foraminifera, Earth-Sci. Rev., 135, 48-58, 2014.

Erez, J.: The Source of Ions for Biomineralization in Foraminifera and Their Implications for Paleoceanographic Proxies, Rev. Mineral. Geochem., 54, 115-149, 2003.

Haynes, J. R.: Foraminifera, John Wiley and Sons, New York, USA, 1981.

Hemleben, C., Erson, O., Berthold, W., and Spindler, M.: Calcification and chamber formation in Foraminifera-a brief overview, in: Biomineralization in lower plants and animals, edited by: Leadbeater, B. S. C. and Riding, R., Clarendon Press, Oxford, UK, 1986.

Hottinger, L.: Illustrated glossary of terms used in foraminiferal research, Carnets Geol., 2, 1-126, 2006.

Hottinger, L. and Dreher, D.: Differentiation of protoplasm in Nummulitidae (foraminifera) from Elat, Red Sea, Mar. Biol., 25, 4161, 1974.

Jepps, M. W.: Studies on Polystomella Lamarck (Foraminifera), J. Mar. Biol. Assoc. UK, 25, 607-666, 1942.

Kunioka, D., Shirai, K., Takahata, N., Sano, Y., Toyofuku, T., and Ujiie, Y.: Microdistribution of $\mathrm{Mg} / \mathrm{Ca}, \mathrm{Sr} / \mathrm{Ca}$, and $\mathrm{Ba} / \mathrm{Ca}$ ratios in Pulleniatina obliquiloculata test by using a NanoSIMS:
Implication for the vital effect mechanism, Geochem. Geophy. Geosy., 7, https://doi.org/10.1029/2006GC001280, 2006.

Leutenegger, S. and Hansen, H. J.: Ultrastructural and radiotracer studies of pore function in foraminifera, Mar. Biol., 54, 11-16, 1979.

Linnaeus, C.: Systema naturae per regna tria naturae, secundum classes, ordinus, genera, species, cum characteribus, differentiis, synonymis, locis. Tomus I, Editio decima, reformata, Impensis Direct, Laurentii Salvii, Holmiae, 1758.

Marszalek, D. S.: Observations on Iridia diaphana, a Marine Foraminifer, J. Protozool., 16, 599-611, 1969.

Myers, E. H.: The life history of Patellina corrugata Williamson, a foraminifer, University of California Press, Oakland, CA, USA, 1935.

Myers, E. H.: Observations on the origin and fate of flagellated gametes in multiple tests of Discorbis (Foraminifera), J. Mar. Biol. Assoc. UK, 24, 201-225, 1940.

Myers, E. H.: Life activities of foraminifera in relation to marine ecology, P. Am. Philos. Soc., 1943, 439-458, 1943.

Nagai, Y., Uematsu, K., Wani, R., and Toyofuku, T.: Reading the Fine Print: Ultra-Microstructures of Foraminiferal Calcification Revealed Using Focused Ion Beam Microscopy, Frontiers in Marine Science, 5, 67, https://doi.org/10.3389/fmars.2018.00067, 2018.

Nehrke, G., Keul, N., Langer, G., de Nooijer, L. J., Bijma, J., and Meibom, A.: A new model for biomineralization and traceelement signatures of Foraminifera tests, Biogeosciences, 10, 6759-6767, https://doi.org/10.5194/bg-10-6759-2013, 2013.

Ní Fhlaithearta, S., Ernst, S. R., Nierop, K. G. J., de Lange, G. J., and Reichart, G.-J.: Molecular and isotopic composition of foraminiferal organic linings, Mar. Micropaleontol., 102, 69-78, 2013.

Nürnberg, D., Bijma, J., and Hemleben, C.: Assessing the reliability of magnesium in foraminiferal calcite as a proxy for water mass temperatures, Geochim. Cosmochim. Ac., 60, 803-814, 1996.

Röttger, R.: Larger foraminifera: reproduction and early stages of development in Heterostegina depressa, Mar. Biol., 26, 5-12, 1974.

Schiebel, R. and Hemleben, C.: Planktic Foraminifers in the Modern Ocean, Springer-Verlag, Berlin Heidelberg, Germany, 2017.

Schultze, M. J. S.: Über den Organismus der Polythalamien (Foraminiferen), nebst Bemerkungen über die Rhizopoden im allgemeinen, Ingelmann, Leipzig, Germany, 1854.

Sliter, W. V.: Bolivina doniezi Cushman and Wickenden in clone culture, Contributions From The Cushman Foundation For Foraminiferal Research, 21, 87-99, 1970.

Spero, H. J.: Ultrastructural examination of chamber morphogenesis and biomineralization in the planktonic foraminifer Orbulina universa, Mar. Biol., 99, 9-20, 1988.

Spindler, M. and Röttger, R.: Chamber formation in large foraminifer Heterostegina depressa (Nummulitidae), Mar. Biol., 18, 146-159, 1973.

Toyofuku, T., Kitazato, H., Kawahata, H., Tsuchiya, M., and Nohara, M.: Evaluation of $\mathrm{Mg} / \mathrm{Ca}$ thermometry in foraminifera: Comparison of experimental results and measurements in nature, Paleoceanography, 15, 456-464, 2000.

Toyofuku, T., De Nooijer, L. J., Yamamoto, H., and Kitazato, H.: Real-time visualization of calcium ion activity in shallow benthic foraminiferal cells using the fluorescent indi- 
cator Fluo-3 AM, Geochem. Geophy. Geosy., 9, Q05005, https://doi.org/10.1029/2007GC001772, 2008.

Toyofuku, T., Suzuki, M., Suga, H., Sakai, S., Suzuki, A., Ishikawa, T., De Nooijer, L. J., Schiebel, R., Kawahata, H., and Kitazato, $\mathrm{H} .: \mathrm{Mg} / \mathrm{Ca}$ and $\delta^{18} \mathrm{O}$ in the brackish shallow-water benthic foraminifer Ammonia "beccarii”, Mar. Micropaleontol., 78, 113-120, 2011.

Toyofuku, T., Matsuo, M. Y., De Nooijer, L. J., Nagai, Y., Kawada, S., Fujita, K., Reichart, G.-J., Nomaki, H., Tsuchiya, M., and Sakaguchi, H.: Proton pumping accompanies calcification in foraminifera, Nat. Commun., 8, 14145, https://doi.org/10.1038/ncomms14145, 2017.

Travis, J. L. and Bowser, S. S.: Reticulopodial Motility, in: The Biology of the Foraminifera, edited by: Lee, J. J. and Anderson, O. R., Academic Press, London, UK, 1991.

Urey, H. C., Lowenstam, H. A., Epstein, S., and McKinney, C. R.: Measurement of paleotemperatures and temperatures of the upper cretaceous of England, Denmark, and the southeastern United States, GSA Bulletin, 62, 399-416, 1951. van Dijk, I., de Nooijer, L. J., and Reichart, G.-J.: Trends in element incorporation in hyaline and porcelaneous foraminifera as a function of $p \mathrm{CO}_{2}$, Biogeosciences, 14, 497-510, https://doi.org/10.5194/bg-14-497-2017, 2017.

Willingham, M. C. and Rutherford, A. V.: The use of OsmiumThiocarbohydrazide- Osmium (OTO) and Ferrocyanide-reduced osmium methods to enhance membrane contrast and preservation in cultured cells, J. Histochem. Cytochem., 32, 4, 455-460, 1984.

Zeebe, R. E. and Sanyal A.: Comparison of two potential strategies of planktonic foraminifera for house building: $\mathrm{Mg}^{2+}$ or $\mathrm{H}^{+}$removal? Geochim. Cosmochim. Ac., 66, 1159-1169, 2002. 